\title{
Orbital Sarcoma cM0 TNM Finding v8
}

National Cancer Institute

\section{Source}

National Cancer Institute. Orbital Sarcoma CMO TNM Finding v8. NCI Thesaurus. Code C140835.

Orbital sarcoma with no distant metastasis. (from AJCC 8th Ed.) 